TITLE:

\title{
Numerical study of comparison of vorticity and passive vectors in turbulence and inviscid flows
}

\section{$\operatorname{AUTHOR}(\mathrm{S})$ :}

Ohkitani, K

\section{CITATION:}

Ohkitani, K. Numerical study of comparison of vorticity and passive vectors in turbulence and inviscid flows. Physical Review E 2002, 65(4): 046304.

\section{ISSUE DATE:}

2002-04

URL:

http://hdl.handle.net/2433/50499

RIGHT:

Copyright 2002 American Physical Society 


\title{
Numerical study of comparison of vorticity and passive vectors in turbulence and inviscid flows
}

\author{
Koji Ohkitani \\ Research Institute for Mathematical Sciences, Kyoto University, Kyoto 606-8502, Japan
}

(Received 28 September 2001; published 28 March 2002)

\begin{abstract}
The nonlinear vortex stretching in incompressible Navier-Stokes turbulence is compared with a linear stretching process of passive vectors (PVs). In particular, we pay special attention to the difference of these processes under long and short time evolutions. For finite time evolution, we confirm our previous finding that the stretching effect of vorticity is weaker than that of general passive vectors for a majority of the initial conditions with the same energy spectra. The above difference can be explained qualitatively by examining the Biot-Savart formula. In order to see to what extent infinitesimal time development explains the above difference, we examine the probability density functions (PDFs) of the stretching rates of the passive vectors in the vicinity of a solution of Navier-Stokes equations. It is found that the PDFs are found to have a Gaussian distribution, suggesting that there are equally many PVs that stretched less and more than the vorticity. This suggests the importance of the vorticity-strain correlation built up over finite time in turbulence. We also discuss the case of Euler equations, where the dynamics of the Jacobian matrix relating the physical and material coordinates is examined numerically. A kind of alignment problem associated with the Cauchy-Green tensor is proposed and studied using the results of numerical simulations. It is found that vorticity tends to align itself with the most compressing eigenvector of the Cauchy-Green tensor. A two-dimensional counterpart of active-passive comparison is briefly studied. There is no essential difference between stretching of vorticity gradients and that of passive scalar gradients and a physical interpretation is given to it.
\end{abstract}

DOI: 10.1103/PhysRevE.65.046304

PACS number(s): 47.27.Ak, 47.10.+g

\section{INTRODUCTION}

By extensive numerical and experimental investigations performed in the past decades, it is understood at least qualitatively that there are coherent vortex structures in turbulence. In the case of homogeneous and isotropic turbulence it is well known that there are tubular vortex structures in the fully developed stage (e.g., see [1]).

The implications of the presence of the intense, localized vortex structures are multifold. First, they are believed to be responsible for transferring mass and heat in actual environment. Second, because compactly supported vortices in totally inviscid flows remain so all the time, localized vortices may be useful to some extent to economize the description of turbulence at high Reynolds numbers [2]. Third, and most fundamental of all, their geometrical structure is believed to influence the net strength of nonlinearity. Such a structure is closely connected with the weakening effects of the apparently quadratic nonlinear stretching, a phenomenon sometimes referred to as depletion of nonlinearity. This notion has been used in a number of similar but different meanings. Originally, it was introduced in [3], where it was called "depression of nonlinearity," to mean that spatial averages of the nonlinear terms such as $\boldsymbol{u} \times \boldsymbol{\omega}$ calculated from turbulence are smaller than those obtained from a random velocity field, which obeys a Gaussian distribution. The buildup of moments including odd-order ones was studied in [4-6] numerically and experimentally. Particularly the "nonlinarity reduction" of the vortex stretching tensor, that is, the observation that $|\boldsymbol{S} \cdot \boldsymbol{\omega}|$ is smaller than the product of norms of $\boldsymbol{S}$ and $\boldsymbol{\omega}$, was studied in detail. It was also shown by a conditional sampling method that such reduction is more significant in vorticity-dominated regions than in strain-dominated regions. In this paper, we also focus on the vortex stretching term $\boldsymbol{\omega} \cdot \boldsymbol{S} \cdot \boldsymbol{\omega}$. To clarify the presence or absence of the BiotSavart constraint $\boldsymbol{S}=\boldsymbol{S}[\boldsymbol{\omega}]$, we compare the vorticity with passive vectors and examine how the constraint makes the vortex stretching term smaller than $\boldsymbol{w} \cdot \boldsymbol{S} \cdot \boldsymbol{w}$, where $\boldsymbol{w}$ is an arbitrary passive vector. It is clear that the direction of vorticity is important here and this point was highlighted mathematically by showing that it can be used to monitor regularity of the Navier-Stokes equations [7] and of the Euler equations [8] (see also [9]).

Recently, we have performed an investigation to illustrate how the stretching effect of vorticity is made weaker by nonlinearity, that is, a kinematic constraint imposed by the BiotSavart law [10]. This was performed by comparing vortex stretching with stretching of passive vectors in turbulence.

The three-dimensional Navier-Stokes equations in vorticity form read

$$
\begin{gathered}
\frac{\partial \boldsymbol{\omega}}{\partial t}+(\boldsymbol{u} \cdot \boldsymbol{\nabla}) \boldsymbol{\omega}=(\boldsymbol{\omega} \cdot \boldsymbol{\nabla}) \boldsymbol{u}+\nu \boldsymbol{\nabla}^{2} \boldsymbol{\omega}, \\
\boldsymbol{\nabla} \cdot \boldsymbol{u}=\boldsymbol{\nabla} \cdot \boldsymbol{\omega}=0, \quad \boldsymbol{\omega}=\boldsymbol{\nabla} \times \boldsymbol{u},
\end{gathered}
$$

and the equations for the passive vectors $w$ are [11]

$$
\begin{gathered}
\frac{\partial \boldsymbol{w}}{\partial t}+(\boldsymbol{u} \cdot \boldsymbol{\nabla}) \boldsymbol{w}=(\boldsymbol{w} \cdot \boldsymbol{\nabla}) \boldsymbol{u}+\nu \boldsymbol{\nabla}^{2} \boldsymbol{w}, \\
\boldsymbol{\nabla} \cdot \boldsymbol{w}=0 .
\end{gathered}
$$

The difference between them is that the stretching term of Eq. (1.1) is constrained by the Biot-Savart relationship whereas that of Eq. (1.3) is not. The Biot-Savart formula 
does a number of things; it makes the vortex stretching term nonlinear and conserve total energy. Thus, Eq. (1.1) has as inviscid invariants

$$
\int|\boldsymbol{u}|^{2} d \boldsymbol{x}, \quad \int \boldsymbol{u} \cdot \boldsymbol{\omega} d \boldsymbol{x},
$$

while Eq. (1.3) has

$$
\int \boldsymbol{u} \cdot \boldsymbol{w} d \boldsymbol{x}, \quad \int \boldsymbol{A} \cdot \boldsymbol{w} d \boldsymbol{x},
$$

where $\boldsymbol{w}=\boldsymbol{\nabla} \times \boldsymbol{A}$. The comparison was performed by using the norm of $\boldsymbol{\omega}$ with that of $\boldsymbol{w}$ on the basis of numerical solutions of the Navier-Stokes equations. We note that this characterization concerns finite- (i.e., long-) time evolution.

The first objective of this paper is to study depletion of nonlinearity in detail by studying the difference between solutions of Eqs. (1.1) and (1.3). The second one is to study infinitesimal-time evolution by examining the stretching rates of both the fields, thereby making clear the distinction between finite- and infinitesimal-time evolutions.

This paper is organized as follows. In Sec. II, we describe detailed analyses of the long-time evolutions of the NavierStokes flows. In Sec. III, we study infinitesimal-time evolution by examining the stretching rates of vorticity and passive vectors (PVs). In Sec. IV, we consider the inviscid flows, that is, flows governed by the Euler equations. Introducing a kind of alignment problem associated with the Cauchy-Green tensor, we provide an alternative characterization of depleted nonlinearity. Section V is devoted to summary and discussion. In Appendix A, we address a similar problem in two dimensions.

\section{FINITE-TIME EVOLUTION: VISCOUS PROBLEM}

We will treat two kinds of initial conditions. The first one, employed in [10], has a compactly supported excitation in the low wave number modes, whose spectrum of energy is given by

$$
E(k)= \begin{cases}k^{2} & \text { only for } \quad k=1,2,3, \\ 0 & \text { otherwise. }\end{cases}
$$

The other one has also localized excitation in wave number space but is not compactly supported,

$$
E(k)=c_{1} k^{2} \exp \left(-k^{2}\right),
$$

where $c_{1}$ is a constant such that $\left\langle|\boldsymbol{\omega}|^{2}\right\rangle / 2=1$. Hereafter, a spatial average over the periodic box $[0,2 \pi]^{3}$ is denoted by brackets throughout this paper.

We review the previous results and consider how they change with increasing Reynolds number for the type-I conditions. We also examine how the results change using a wider class of initial conditions of the type II.

\section{A. Higher Reynolds numbers}

Clearly, if the initial conditions $\boldsymbol{\omega}(\boldsymbol{x}, 0)$ and $\boldsymbol{w}(\boldsymbol{x}, 0)$ are exactly the same, then the solutions are the same by the
TABLE I. Numerical parameters for type-I conditions.

\begin{tabular}{lcccc}
\hline \hline$\nu$ & $5 \times 10^{-3}$ & $2.5 \times 10^{-3}$ & $1.5 \times 10^{-3}$ & $1 . \times 10^{-3}$ \\
$d t$ & $5 \times 10^{-3}$ & $5 \times 10^{-3}$ & $2 . \times 10^{-3}$ & $1 . \times 10^{-3}$ \\
$N$ & 128 & 128 & 256 & 256 \\
\hline \hline
\end{tabular}

uniqueness argument for the solutions of Eq. (1.1). Concerning Eqs. (1.1) and (1.3), a question raised in [10] is how can their subsequent time evolution differ, if the initial conditions are similar but not exactly the same in the sense that they only share the Fourier spectrum of energy.

For the initial condition of type I, we compute using four different values of kinematic viscosity $\nu=5 \times 10^{-3}, 2.5$ $\times 10^{-3}, 1.5 \times 10^{-3}$, and $1 \times 10^{-3}$. The computations are performed by a standard pseudospectral method with aliasing errors removed by the $2 / 3$ law. Thus, the maximum wave number is $N / 3$ for calculations with grid points $N^{3}$. Time marching is performed by the Runge-Kutta method. When we solve Eqs. (1.1) and (1.3) simultaneously, computations with $N=128$ are done in double precision arithmetic and those with $N=256$ in single precision, because a large amount of memory is required to solve them simultaneously. We have checked that no essential differences in the time evolution were found between computations in single and double precision for $N=128$ computations. We have also checked that no differences were found between computations of Navier-Stokes flows in single and double precision at $N=256$ resolution. The numerical parameters are summarized in Table I. The Reynolds numbers based on the Taylor microscale are $R_{\lambda}=45.8,67.3,86.3$, and 101.3 at $t=4$ and $R_{\lambda}=33.3,45.7,55.1$, and 66.1 at $t=6$.

We show in Fig. 1(a) time evolution of the energy dissi-
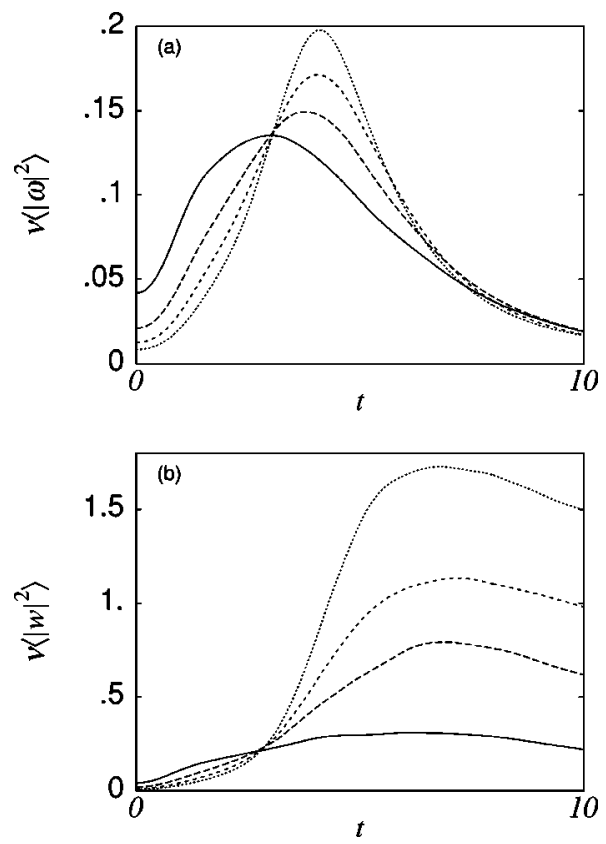

FIG. 1. Time evolution of (a) the energy dissipation rate $\epsilon(t)$ and (b) a corresponding quantity $\epsilon_{\mathrm{PV}}(t)$ for PVs. The values of viscosity are $\nu=5 \times 10^{-3}$ (solid), $2.5 \times 10^{-3}$ (dashed), $1.5 \times 10^{-3}$ (short-dashed), and $1.0 \times 10^{-3}$ (dotted). 

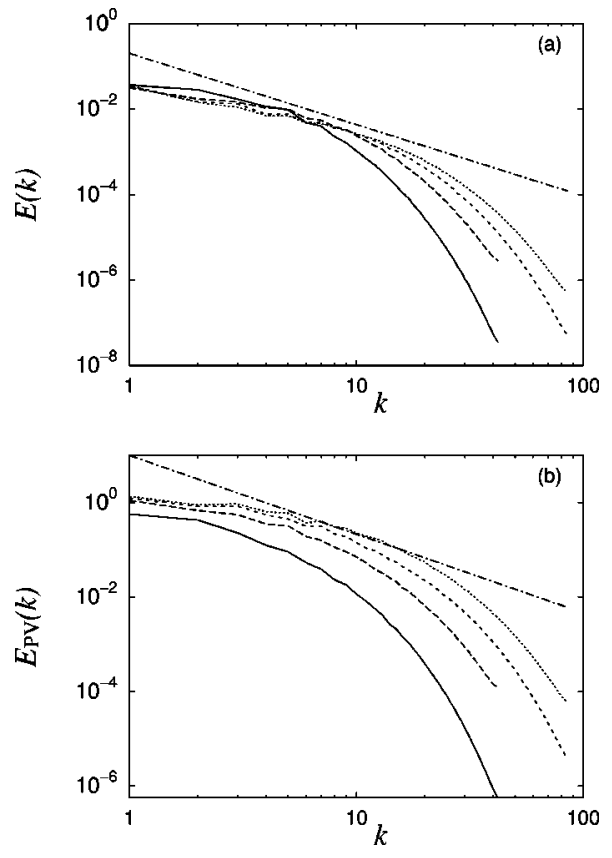

FIG. 2. The energy spectra (a) $E(k)$ and (b) $E_{\mathrm{PV}}(k)$. The line types are the same as in Fig. 1.

pation rate

$$
\epsilon(t)=\nu\left\langle|\boldsymbol{\omega}|^{2}\right\rangle
$$

for the four different values of viscosity. Except for the case of the largest value of viscosity, the peak value changes only a little, that is, less than $25 \%$, while the value of viscosity is changed by a factor of 2.5 . The time of peak dissipation rates does not change significantly either. This does not imply, but is consistent with a property of the energy dissipation rate that behaves independent of the viscosity in the limit $\nu \rightarrow 0$.

A corresponding spatial average for $\boldsymbol{w}$,

$$
\epsilon_{P V}(t)=\nu\left\langle|w|^{2}\right\rangle
$$

is shown in Fig. 1(b). In contrast to the energy dissipation rate, it is remarkable that this quantity keeps increasing at its peak value as viscosity is decreased. It shows no trend of tending to a limiting behavior as $\nu \rightarrow 0$. The difference in the limiting behavior between Eqs. (2.3) and (2.4) is the first evidence that the passive vector $\boldsymbol{w}$ is more intensely stretched than $\boldsymbol{\omega}$. As mentioned above, the only difference between Eqs. (1.1) and (1.3) is the presence of energy conservation for the former. So, the above difference in behavior in the limit of small viscosity comes from the fact that Eq. (2.3) is a well-defined physically meaningful quantity, whereas Eq. (2.4) is not (see Table III). This result strengthens the finding reported in [10] and can be interpreted to indicate that under finite-time evolution, the $\boldsymbol{\omega}-\boldsymbol{S}$ correlation is developed more strongly than the $\boldsymbol{w}-\boldsymbol{S}$ correlation.

In Fig. 2(a) we show the energy spectrum

$$
E(k)=\frac{1}{2} \sum_{k \leqslant|\boldsymbol{k}|<k+1}|\tilde{\boldsymbol{u}}(\boldsymbol{k})|^{2}
$$

at time $t=10$ after the turbulence has fully developed. This figure shows that at higher Reynolds number the shape of the spectrum is compatible with a short Kolmogorov's 5/3 range, while it is hard to detect it clearly because of rather limited resolution used here. In comparison, we show in Fig. 2(b) a corresponding spectrum for passive vector

$$
E_{\mathrm{PV}}(k)=\frac{1}{2} \sum_{k \leqslant|\boldsymbol{k}|<k+1}|\widetilde{\boldsymbol{A}}(\boldsymbol{k})|^{2} .
$$

At first glance this figure does not look very different from the previous one. By examining them more carefully we find that the amplitude is much larger than that of Fig. 2(a) and a power law range is less clearly seen here. Again, this stems from the lack of energy conservation in the stretching term in Eq. (1.3).

Next, we examine the time evolution of the statistical properties on the alignment process of the vorticity and passive vectors with the rate-of-strain tensor and compare them with the results of $[5,6]$.

In Fig. 3 we show the probability density functions (PDFs) of the cosines of the angle between the vorticity and each eigenvector of $S$. As is well known, a strong preference of the vorticity to align itself with the intermediate eigenvector is observed. It should be noted that this property is observed before the time of maximum enstrophy $t \approx 3$ and persists during the decaying process. In Fig. 4 we show the corresponding PDFs of the angle between the passive vector and each eigenvector $S$. Unlike Fig. 3 the property changes in time. In the early stage $(t=2)$, passive vectors strongly align themselves with the intermediate eigenvector, just like the vorticity. However, after the time of maximum enstrophy the preference to the intermediate strain is getting weaker while the preference to the most expanding strain stronger. At $t=10$ the PDFs associated with the largest and the intermediate strains almost collapse. As time goes on, the stretching effect is enhanced by redirecting the passive vector to the most expanding strain eigenvector.

There are a number of investigations on the stretching of material line elements, that is, passive vectors without diffusivity (see, e.g., [12].) It was reported in [13] that they have a tendency to align themselves with the most expanding strain eigenvector (see $[4,5,14]$ for relevant discussions). We note that an accurate estimate of material line stretching requires special care of numerical treatment [15]. We also note that a comparison was made of active and passive vectors in a different formulation in [16].

We consider the stretching rates of vorticity $\alpha$ and that of passive vector $\beta$, which are defined by

$$
\alpha(\boldsymbol{x}, t)=\frac{\boldsymbol{\omega} \cdot \boldsymbol{S} \cdot \boldsymbol{\omega}}{|\boldsymbol{\omega}|^{2}}
$$

and

$$
\beta(x, t)=\frac{w \cdot S \cdot w}{|w|^{2}}
$$



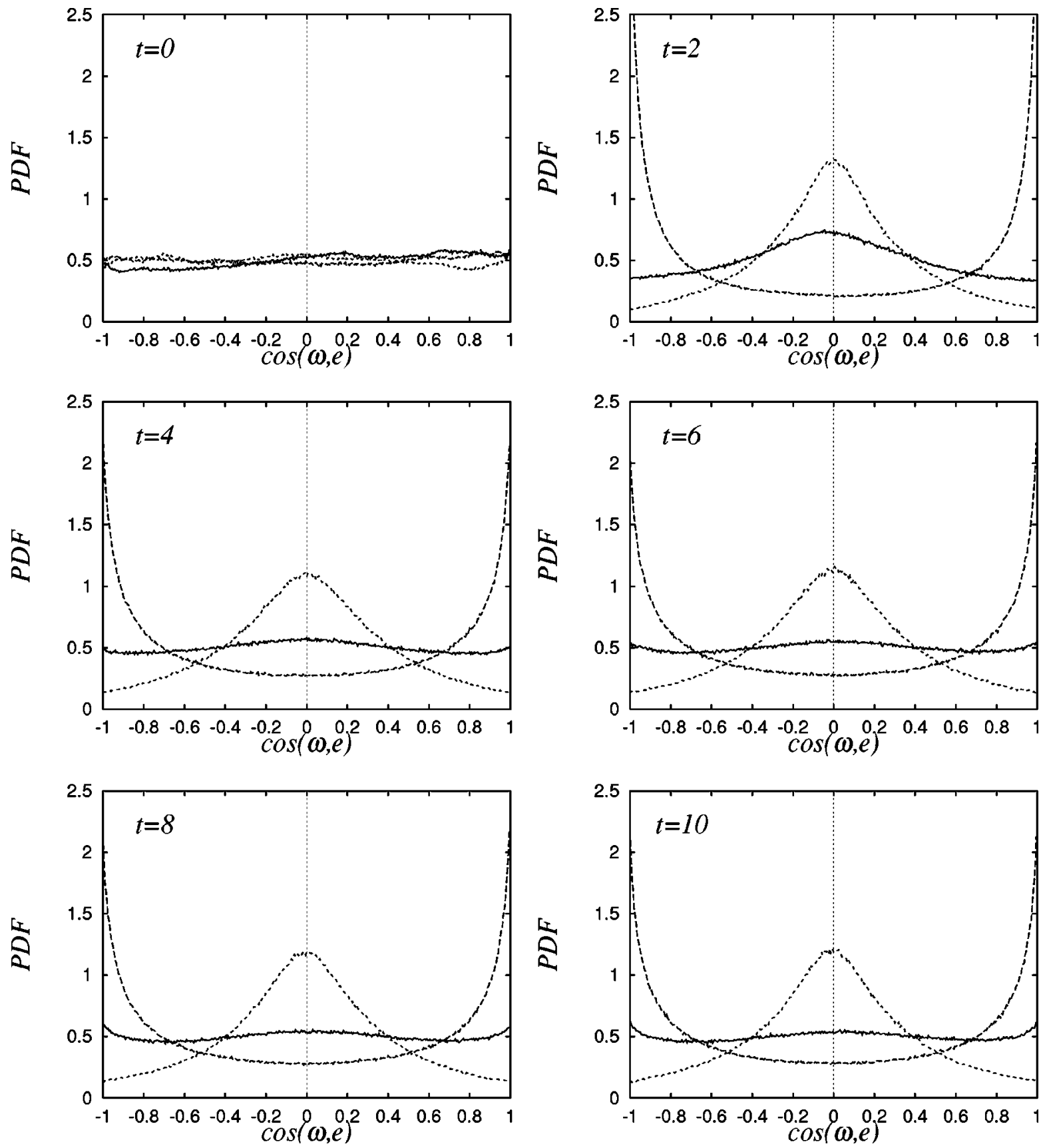

FIG. 3. The PDFs of the cosines that the angle $\boldsymbol{\omega}$ makes with $\boldsymbol{e}_{1}$ (solid), $\boldsymbol{e}_{2}$ (dashed), and $\boldsymbol{e}_{3}$ (dotted), at times $t=0,2,4,6,8$, and 10 .

Their PDFs are shown in Fig. 5(a) in a normalized form as $\tilde{\alpha}=\alpha / \sqrt{\left\langle|\boldsymbol{\omega}|^{2}\right\rangle}$ and $\widetilde{\beta}=\beta / \sqrt{\left\langle|\boldsymbol{\omega}|^{2}\right\rangle}$, together with that of the three eigenvalues of the rate-of-strain tensor $\quad \tilde{\lambda_{1}}=\lambda_{1} / \sqrt{\left\langle|\boldsymbol{\omega}|^{2}\right\rangle}, \quad \tilde{\lambda_{2}}=\lambda_{2} / \sqrt{\left\langle|\boldsymbol{\omega}|^{2}\right\rangle}, \quad$ and $\tilde{\lambda_{3}}$ $=\lambda_{3} / \sqrt{\left\langle|\boldsymbol{\omega}|^{2}\right\rangle}$. Not surprisingly, the PDF of $\tilde{\alpha}$ is close to that of $\tilde{\lambda_{2}}$. It was unexpected, however, that the PDF of $\widetilde{\beta}$ is also fairly close to $\lambda_{2}$. In Fig. 5(b) we show an enlarged plot of $\alpha$ and $\widetilde{\beta}$. Indeed, the PDF of the stretching rate $\widetilde{\beta}$ of $\mathrm{PV}$ is shifted positively compared with that of the vorticity. It should be noted that the slight difference in the PDFs leads to a big difference in the long-time evolution. This is the second quantitative characterization that PVs are stretched more intensely than the vorticity.
It is of interest to examine how regions with intense $|\boldsymbol{\omega}|$ and $|\boldsymbol{w}|$ look like in physical space. We show in Fig. 6(a) the isosurface plots of $|\boldsymbol{\omega}|^{2}$ and $|\boldsymbol{w}|^{2}$ at time $t=2$, well before the enstrophy attains its maximum. Even in this early stage lots of vorticity tubes are already formed in $|\boldsymbol{\omega}|$. On the other hand, in $|\boldsymbol{w}|^{2}$, layerlike structures are apparently predominant. In Fig. 6(b) is shown a similar plot at $t=6$ in the fully developed stage. It is hard to distinguish $|\boldsymbol{\omega}|^{2}$ and $|\boldsymbol{w}|^{2}$ by solely looking at these pictures. Note that the threshold is chosen such that the volume enclosed is $1 \%$ for both fields. It is possible to distinguish the isosurfaces of $|\boldsymbol{\omega}|^{2}$ from those of $|\boldsymbol{w}|^{2}$ in the early stage, but not in the fully developed stage. Recalling the fact that their dynamics are markedly 

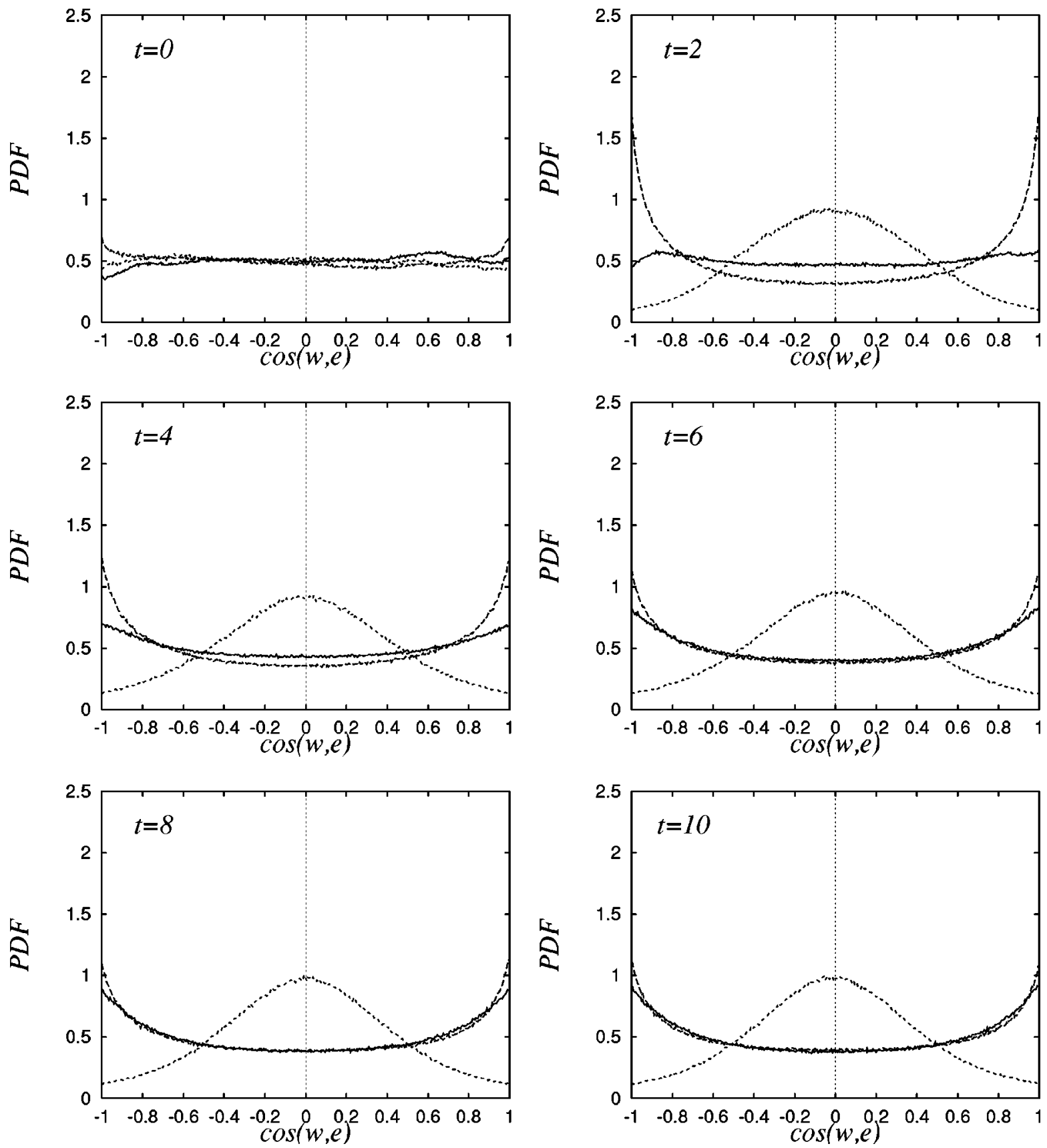

FIG. 4. The PDFs of the cosines that the angle $\boldsymbol{w}$ makes with $\boldsymbol{e}_{1}$ (solid), $\boldsymbol{e}_{2}$ (dashed), and $\boldsymbol{e}_{3}$ (dotted) at times $t=0,2,4,6,8$, and 10 .

different, the similarity of isosurface characteristics in turbulence is rather unexpected.

\section{B. A wider class of initial conditions}

In the above we have seen a big difference between vorticity and passive vectors in turbulence starting from an initial condition and that this difference is pronounced at higher Reynolds numbers. We should ask whether such a difference is accidental, i.e., specific to this particular initial condition, or ubiquitous over a broad range of initial conditions.

Under the evolution of Navier-Stokes equations over finite time, correlation builds up both between the vorticity and the rate of strain and between passive vectors and the rate of strain. This point has been studied in a different manner in [3]. The latter correlation $\boldsymbol{w}-\boldsymbol{S}$ is stronger than the former $\boldsymbol{\omega}-S$ in the sense that $\epsilon_{P V}$ increases whereas $\epsilon$ remains finite, as the value of viscosity is decreased. This suggests that $\epsilon_{P V}$ increases without bound whereas $\epsilon$ remains finite in the limit of vanishing viscosity. Further numerical experiments will be necessary to confirm this trend at higher Reynolds numbers.

We generate an initial condition of type II, which is given by Eq. (2.2). We then generate the initial condition for $\boldsymbol{w}$ by completely destroying the phases of the Fourier coefficients of $\boldsymbol{\omega}$ as

$$
\tilde{\boldsymbol{w}}(\boldsymbol{k})=\exp (i \theta(\boldsymbol{k})) \tilde{\boldsymbol{\omega}}(\boldsymbol{k}),
$$



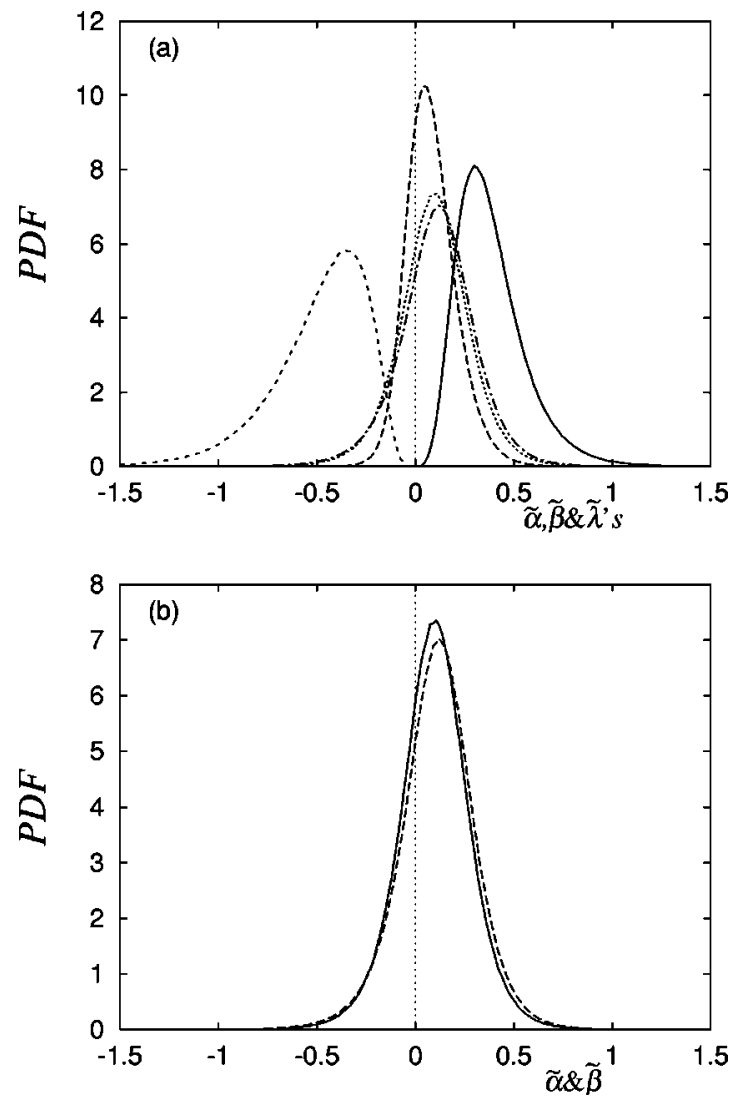

FIG. 5. The PDFs of (a) the stretching rates $\widetilde{\alpha}$ (dotted), $\widetilde{\beta}$ (dashdotted), and three eigenvalues $\tilde{\lambda_{1}}$ (solid), $\tilde{\lambda_{2}}$ (dashed), and $\tilde{\lambda_{3}}$ (short-dashed) of the rate-of-strain tensor and (b) the enlargement for $\tilde{\alpha}$ (solid) and $\widetilde{\beta}$ (dashed).

where $\theta(\boldsymbol{k})$ denotes uniform pseudorandom numbers in $[0,2 \pi]$. It is clear that the solenoidal condition $\boldsymbol{\nabla} \cdot \boldsymbol{w}=0$ is satisfied.

We show in Fig. 7(a) the time evolution of the total enstrophy $\left\langle|\boldsymbol{\omega}|^{2}\right\rangle / 2$ together with the total passive vector variance $\left\langle|\boldsymbol{w}|^{2}\right\rangle / 2$ for ten different initial conditions of $\boldsymbol{w}$. It is remarkable that in all the cases the norm of $\boldsymbol{w}$ grow faster than the enstrophy in their long-time evolution. For the class of initial conditions, we confirm that the vorticity grows faster than passive vectors in the case of a wider class of initial conditions.

Then, what happens if we choose the initial conditions for $\boldsymbol{w}$ in an immediate vicinity of $\boldsymbol{\omega}$. To check this point we performed computations, starting from yet another ten different initial conditions with $\theta(\boldsymbol{k}) \in[0,0.05 \times 2 \pi]$, that is, the phases randomized only slightly by $5 \%$. As we can see in Fig. 7(b) the passive vector variances deviate from the enstrophy for these class of initial conditions and the majority of them grow faster than the enstrophy at short times. Actually, if we examine Fig. 7(b) more carefully, we see that for two cases out of ten initial conditions, the passive vector variance becomes a little bit smaller than the enstrophy in the early stage of time development.

We show one such example in Fig. 8(a) in detail. We have checked in Fig. 8(b) that even in the inviscid case the passive vector variance is still smaller than the enstrophy. This means that it is not the effect of finite Reynolds number that

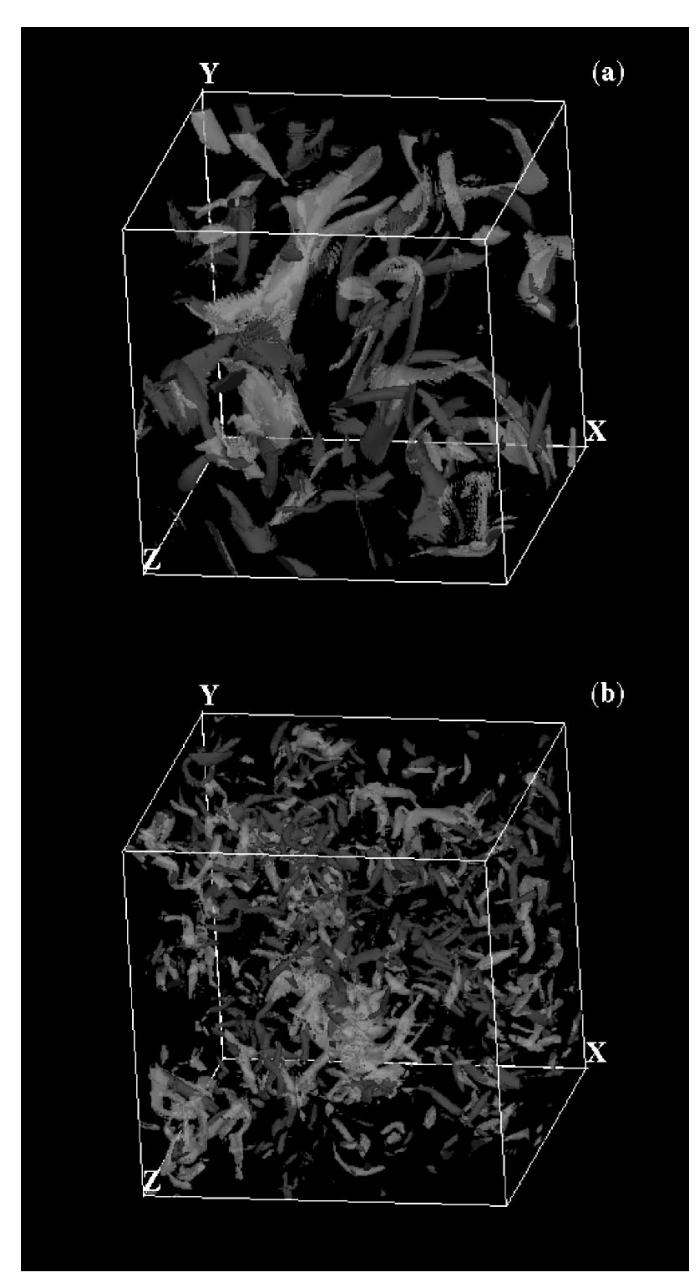

FIG. 6. Isosurface plots of $|\boldsymbol{\omega}|^{2}$ (darker) and $|\boldsymbol{w}|^{2}$ (lighter). (a) At $t=2$ in the early stage and (b) at $t=6$ in the fully developed stage. The full computational box $[0,2 \pi]^{3}$ is shown. The thresholds are chosen such that the total volume enclosed inside the isosurfaces is $1 \%$ of the whole box in each case.

makes the enstrophy larger than the passive vector variance.

We summarize our numerical results regarding finite-time evolution as follows: for a large class of solenoidal initial conditions, high-Reynolds-number turbulence satisfies

$$
\left\langle|\boldsymbol{w}(\boldsymbol{x}, t)|^{2}\right\rangle>\left\langle|\boldsymbol{\omega}(\boldsymbol{x}, t)|^{2}\right\rangle
$$

in the long run, provided $\boldsymbol{w}(\boldsymbol{x}, 0)$ shares the Fourier spectra with $\boldsymbol{\omega}(\boldsymbol{x}, 0)$, but is substantially different from $\boldsymbol{\omega}(\boldsymbol{x}, 0)$. This property does not hold if we take initial conditions $\boldsymbol{w}(\boldsymbol{x}, 0)$ very close to $\boldsymbol{\omega}(\boldsymbol{x}, 0)$. While there are some cases where the enstrophy becomes larger than the variance of PV at some stage of time development, the overall trend of dominance of growth in PVs over that of the vorticity is remarkable.

Moreover, on top of making them share the spectrum with $\boldsymbol{\omega}(\boldsymbol{x}, 0)$, if we impose restrictions on $\boldsymbol{w}(\boldsymbol{x}, 0)$ further, it may be possible that we make

$$
\left\langle|\boldsymbol{w}(\boldsymbol{x}, t)|^{2}\right\rangle \geqslant\left\langle|\boldsymbol{\omega}(\boldsymbol{x}, t)|^{2}\right\rangle
$$



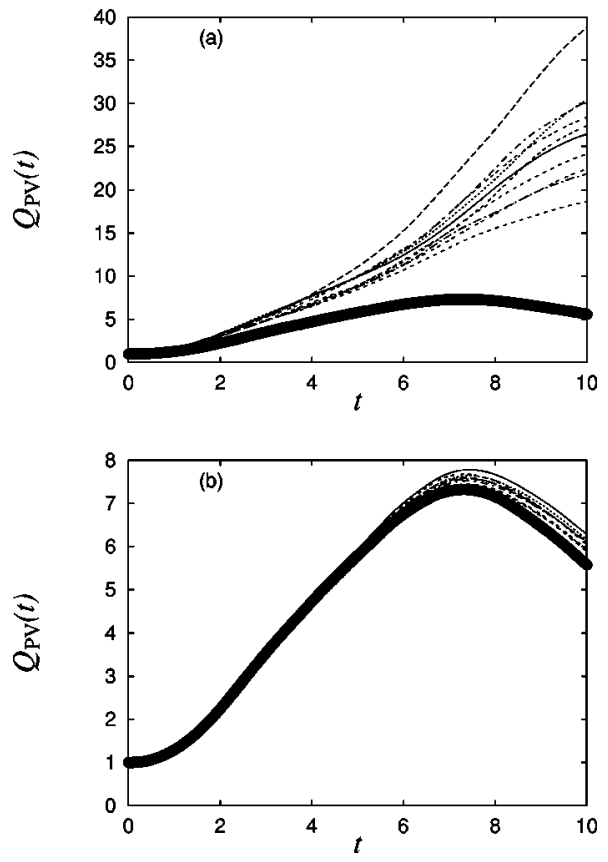

FIG. 7. Time evolution of $Q_{\mathrm{PV}}(t)$ for ten different initial conditions (thin lines) and that of $Q(t)$ (a thick line), where the phases of $\boldsymbol{w}$ are (a) completely and (b) slightly destroyed.

at short times. See, Appendix B for an example constructed using the Taylor-Green vortex. For general incompressible flows, it is not known, but worthwhile to study under what conditions (2.11) holds valid.

\section{A qualitative explanation}

At a crude level, we can explain the above difference as follows. For simplicity we work in the infinite space bound-
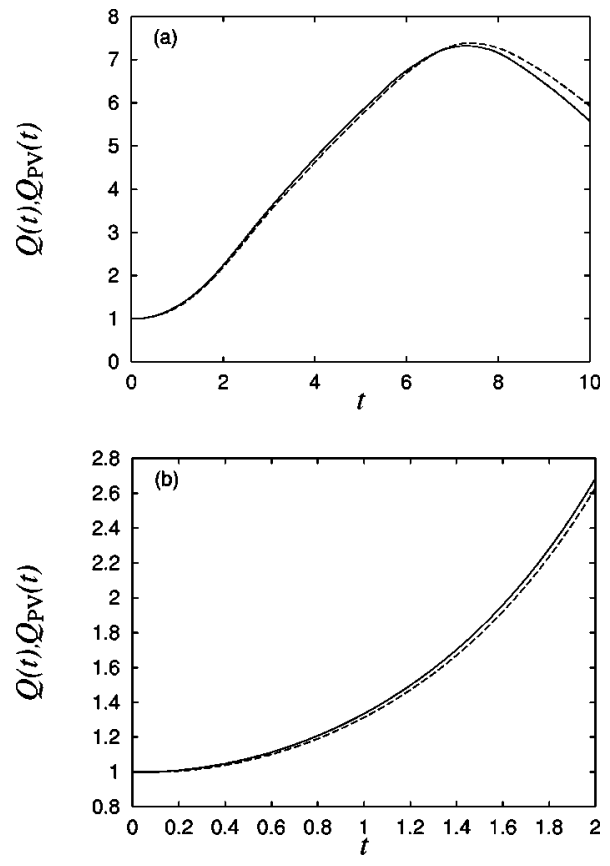

FIG. 8. An example of a comparison where $Q(t)$ (solid) becomes larger than $Q_{\mathrm{PV}}(t)$ (dashed) at some stage; (a) the viscous case and (b) the early stage inviscid case. ary condition where the fluid is at rest at infinity. Using the Biot-Savart formula we have [9]

$$
\alpha(\boldsymbol{x})=\frac{3}{4 \pi} \mathrm{P} \int[\hat{\boldsymbol{y}} \cdot \boldsymbol{\xi}(\boldsymbol{x})][\hat{\boldsymbol{y}} \cdot\{\boldsymbol{\omega}(\boldsymbol{x}+\boldsymbol{y}) \times \boldsymbol{\xi}(\boldsymbol{x})\}] \frac{d \mathbf{y}}{|\mathbf{y}|^{3}},
$$

where $\hat{y} \equiv \boldsymbol{y} /|\boldsymbol{y}|, \quad \xi \equiv \boldsymbol{\omega}(\boldsymbol{x}) /|\boldsymbol{\omega}(\boldsymbol{x})|$. Similarly, we find

$$
\beta(\boldsymbol{x})=\frac{3}{4 \pi} \mathrm{P} \int[\hat{\boldsymbol{y}} \cdot \boldsymbol{\eta}(\boldsymbol{x})][\hat{\boldsymbol{y}} \cdot\{\boldsymbol{\omega}(\boldsymbol{x}+\boldsymbol{y}) \times \boldsymbol{\eta}(\boldsymbol{x})\}] \frac{d \mathbf{y}}{|\mathbf{y}|^{3}},
$$

where $\boldsymbol{\eta} \equiv \boldsymbol{w}(\boldsymbol{x}) /|\boldsymbol{w}(\boldsymbol{x})|$ and $\mathrm{P}$ is the principal value.

In Eq. (2.12), $\boldsymbol{\omega}(\boldsymbol{x}+\boldsymbol{y}) \times \boldsymbol{\xi}(\boldsymbol{x})$ is likely to be small and $\boldsymbol{y}$ is small if the direction of $\boldsymbol{\omega}(\boldsymbol{x})$ is smooth. On the other hand in Eq. (2.13) there is no reason for $\boldsymbol{\omega}(\boldsymbol{x}+\boldsymbol{y}) \times \boldsymbol{\eta}(\boldsymbol{x})$ to become small for small $\boldsymbol{y}$ even if the direction of $\boldsymbol{w}(\boldsymbol{x})$ is smooth, because the correlation between $\boldsymbol{\omega}(\boldsymbol{x})$ and $\boldsymbol{w}(\boldsymbol{x})$ remains weak. This lack of depleting mechanism qualitatively accounts for the slower growth of the vorticity compared with passive vectors.

\section{INFINITESIMAL TIME DEVELOPMENT}

In the above we have seen that the difference in the behavior of the vorticity and PVs can be partially and qualitatively explained on the basis of analysis of the Biot-Savart formula. However, we should keep in mind that this formula is kinematic and in this sense incorporates only infinitesimal evolution of the vorticity equation, as represented by its right-hand side.

We ask in this section whether we can characterize the significant difference found in long-time evolution solely by studying the infinitesimal-time development. To this end, we compare the PDFs of the growth rates of passive vector variance with the growth rates of total enstrophy. The passive vectors are taken in the vicinity of the vorticity in the threedimensional incompressible vector space. There are two different kinds of ways to specify the vorticity around which PDFs are generated. (1) As fully developed turbulence and (2) as one of the smooth random initial conditions. This analysis was carried out for the calculation with $128^{3}$ grid points because of the computational task to produce the PDF of growth rates is formidable. The low resolution poses no restriction on (2) but limits the Reynolds number to low values for (1). Needless to mention, the former choice is dynamically influenced by the Navier-Stokes equations, but the latter one has no such dynamical property. It is of interest to study how this difference in the choice of the vorticity leads to the statistical property of PVs around it.

The growth rate $\sigma_{\omega}$ of the total enstrophy is defined as

$$
\sigma_{\omega}(t)=\frac{1}{\left\langle|\boldsymbol{\omega}|^{2}\right\rangle} \frac{d}{d t}\left\langle|\boldsymbol{\omega}|^{2}\right\rangle=2 \frac{\langle\boldsymbol{\omega} \cdot \boldsymbol{S} \cdot \boldsymbol{\omega}\rangle}{\left\langle|\boldsymbol{\omega}|^{2}\right\rangle},
$$

whereas that of passive vector variance as 


$$
\sigma_{\mathrm{PV}}(t)=\frac{1}{\left\langle|\boldsymbol{w}|^{2}\right\rangle} \frac{d}{d t}\left\langle|\boldsymbol{w}|^{2}\right\rangle=2 \frac{\langle\boldsymbol{w} \cdot \boldsymbol{S} \cdot \boldsymbol{w}\rangle}{\left\langle|\boldsymbol{w}|^{2}\right\rangle} .
$$

We consider passive vectors in the vicinity of $\boldsymbol{\omega}$ in solenoidal vector space, under the condition $\left\langle|\boldsymbol{w}|^{2}\right\rangle=\left\langle|\boldsymbol{\omega}|^{2}\right\rangle$.

In practice, we generate $\boldsymbol{w}$ as

$$
\tilde{\boldsymbol{w}}(\boldsymbol{k})=\exp [i \theta(\boldsymbol{k})] \tilde{\boldsymbol{\omega}}(\boldsymbol{k}),
$$

where $\theta(\boldsymbol{k}) \in[0, \epsilon \times 2 \pi]$. We have tried a number of different values of $\epsilon$, which are $\epsilon=1$ (the completely randomized case), 0.7, 0.5, 0.2, 0.1, and 0.05. For each choice of $\epsilon$, 50000 realizations are used to produce PDFs.

Because $\epsilon$ is a measure of scrambledness, we have

$$
\overline{\langle\boldsymbol{w} \cdot \boldsymbol{S} \cdot \boldsymbol{w}\rangle} \rightarrow\langle\boldsymbol{\omega} \cdot \boldsymbol{S} \cdot \boldsymbol{\omega}\rangle \quad \text { as } \quad \epsilon \rightarrow 0,
$$

where the bar denotes ensemble average and

$$
\overline{\langle\boldsymbol{w} \cdot \boldsymbol{S} \cdot \boldsymbol{w}\rangle} \rightarrow 0 \quad \text { as } \quad \epsilon \rightarrow 1 .
$$

Let us consider the PDF $P(X)$ of

$$
X \equiv \frac{2}{\sigma_{X}}\left(\frac{\langle w \cdot S \cdot w\rangle}{\left\langle|w|^{2}\right\rangle}-\frac{\overline{w \cdot S \cdot w\rangle}}{\left\langle|w|^{2}\right\rangle}\right),
$$

where $\sigma_{X}$ is the standard deviation of $X$

$$
\sigma_{x}=2 \sqrt{\left(\frac{\langle w \cdot S \cdot w\rangle}{\left\langle|w|^{2}\right\rangle}-\frac{\langle\bar{w} \cdot \boldsymbol{S} \cdot \boldsymbol{w}\rangle}{\left\langle|w|^{2}\right\rangle}\right)^{2}} .
$$

Note that $P(X)$ has zero mean and unit variance.

We show in Fig. 9(a) the PDF $P(X)$ for the case (1) and Fig. 9(b) for the case (2). It should be noted that the PDFs show a normal (Gaussian) distribution, not only for (2) but also for (1). The normal distribution was expected for (2) because the vorticity is just one of the initial conditions and therefore contains no dynamical information of the NavierStokes equations. However, for (1) where the vorticity is taken from the fully developed turbulence, it was anticipated that the PDF of the stretching rates of PVs might have a skewness indicating greater stretching rates than that of the enstrophy.

To make the data analysis more quantitatively, we examine two statistical quantities, the average

$$
m=\frac{\overline{\langle\boldsymbol{w} \cdot \boldsymbol{S} \cdot \boldsymbol{w}\rangle}}{\langle\boldsymbol{\omega} \cdot \boldsymbol{S} \cdot \boldsymbol{\omega}\rangle}
$$

and the standard deviation

$$
\sigma=\sqrt{\frac{\overline{(\langle w \cdot S \cdot w\rangle-\overline{\langle w \cdot S \cdot w\rangle})^{2}}}{\left(\langle\omega \cdot S \cdot \omega\rangle-\langle\overline{w \cdot S \cdot w\rangle})^{2}\right.}}
$$

both have been nondimensionalized against the enstrophy growth rate. The results are summarized in Table II. Both for turbulence and a random field, all in all, $m$ and $\sigma$ become
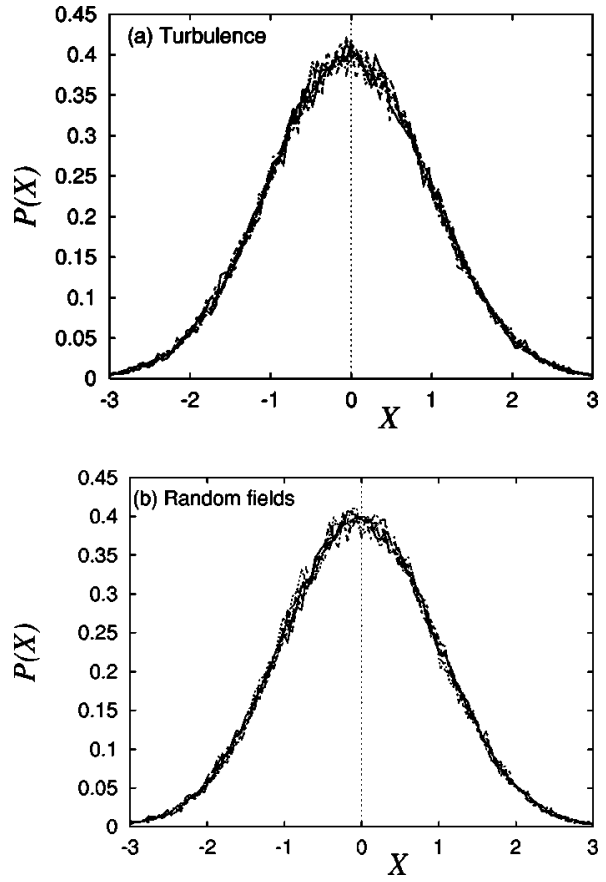

FIG. 9. The PDFs of stretching rates of PVs for $\epsilon=0.05,0.10$, $0.20,0.50,0.70,1.0$ : around (a) $\boldsymbol{\omega}$, which is a solution of the Navier-Stokes equations, and (b) $\boldsymbol{\omega}$, which is a random field. A standard normal distribution, also depicted as a solid curve, is virtually invisible because the PDFs are so close to it in both cases.

smaller for larger values of $\epsilon$. Two features that distinguish turbulence and a random field should be noted. First, the mean value $m$ decays more quickly for the case of a random field than for the case of turbulence. This can be attributed to the presence of the vorticity-strain correlation in turbulence. Second, $\sigma$ for random fields are much larger than that for turbulence because the denominator becomes small for small $\epsilon$.

The present result suggests that it is virtually impossible to explain the big difference in the behavior of the vorticity and PVs in their long-time evolution by solely looking at their infinitesimal-time development.

\section{THE INVISCID PROBLEM}

We have seen that it is necessary to take into account the long-time evolution to explain the big difference between the vorticity and passive vector. The conventional alignment

TABLE II. Statistics of growth rates of PVs.

\begin{tabular}{ccccc}
\hline \hline & Turbulence & & Random fields & \\
\hline$\epsilon$ & $m$ & $\sigma$ & $m$ & $\sigma$ \\
\hline 0.05 & $5.7 \times 10^{-2}$ & $7.6 \times 10^{-2}$ & 1.0 & 2.4 \\
0.10 & $5.6 \times 10^{-2}$ & $4.8 \times 10^{-2}$ & 1.0 & 7.5 \\
0.20 & $5.2 \times 10^{-2}$ & $3.3 \times 10^{-2}$ & $7.7 \times 10^{-1}$ & 2.6 \\
0.50 & $2.5 \times 10^{-2}$ & $1.5 \times 10^{-2}$ & $-1.0 \times 10^{-1}$ & 1.2 \\
0.70 & $8.2 \times 10^{-3}$ & $9.3 \times 10^{-3}$ & $-8.0 \times 10^{-3}$ & 1.4 \\
1.00 & $7.6 \times 10^{-7}$ & $7.6 \times 10^{-3}$ & $6.3 \times 10^{-3}$ & 1.4 \\
\hline \hline
\end{tabular}


analysis, that is, the comparison of the vorticity with the eigenvectors of the rate-of-strain tensor is not sufficiently useful in this respect, because it is relevant to instantaneoustime development only. In order to characterize the vortex stretching process better we will consider in this section an alternative alignment problem.

\section{A. Numerical methods}

We are interested in the Jacobian matrix relating spatial and material coordinates,

$$
J_{i j}=\frac{\partial x_{i}}{\partial a_{j}} \quad(i, j=1,2,3) .
$$

Here we restrict ourselves to the consideration of inviscid flows. The Jacobian matrix satisfies the following equations:

$$
\frac{\partial J_{i j}}{\partial t}+u_{k} \frac{\partial J_{i j}}{\partial x_{k}}=\frac{\partial u_{i}}{\partial x_{k}} J_{k j},
$$

where summation is implicit on repeated indices. To treat it numerically, it is useful to note that

$$
\frac{D}{D t} \frac{\partial J_{i j}}{\partial x_{i}}=0 \quad \text { for } \quad j=1,2,3 \text {, }
$$

which can be shown readily from Eq. (4.2). Therefore, if we take as usual $J_{i j}=\delta_{i j}$ at $t=0$, then we have

$$
\frac{\partial J_{i j}}{\partial x_{i}} \equiv 0
$$

at later times. We may thus regard each column of $J_{i j}$ as a solenoidal vector, which allows us to reduce memory size and computations in their calculations. Introducing

$$
\left(\boldsymbol{W}_{j}\right)_{i} \equiv J_{i j}
$$

we can write

$$
\frac{\partial \boldsymbol{W}_{j}}{\partial t}=\boldsymbol{\nabla} \times\left(\boldsymbol{u} \times \boldsymbol{W}_{j}\right) \quad \text { for } \quad j=1,2,3 .
$$

On top of the vorticity equation, we have three more similar equations to be solved simultaneously.

\section{B. An alternative alignment}

Let us recall the Cauchy formula

$$
\boldsymbol{\omega}(a, t)=J \omega_{0}(a),
$$

which is a first integral of the Euler equations. Using it, we can express the enstrophy in material coordinates as

$$
|\boldsymbol{\omega}(a, t)|^{2}=\boldsymbol{\omega}_{0}(a)^{T} \boldsymbol{J}^{T} \boldsymbol{J} \boldsymbol{\omega}_{0}(a) .
$$

Here the matrix $\boldsymbol{J}^{T} \boldsymbol{J}$ is symmetric and is a positive-definite real matrix, which is known as the Cauchy-Green tensor, see, e.g., [17]. It has three eigenvalues $\alpha, \beta, \gamma \quad(\alpha \geqslant \beta \geqslant \gamma>0)$
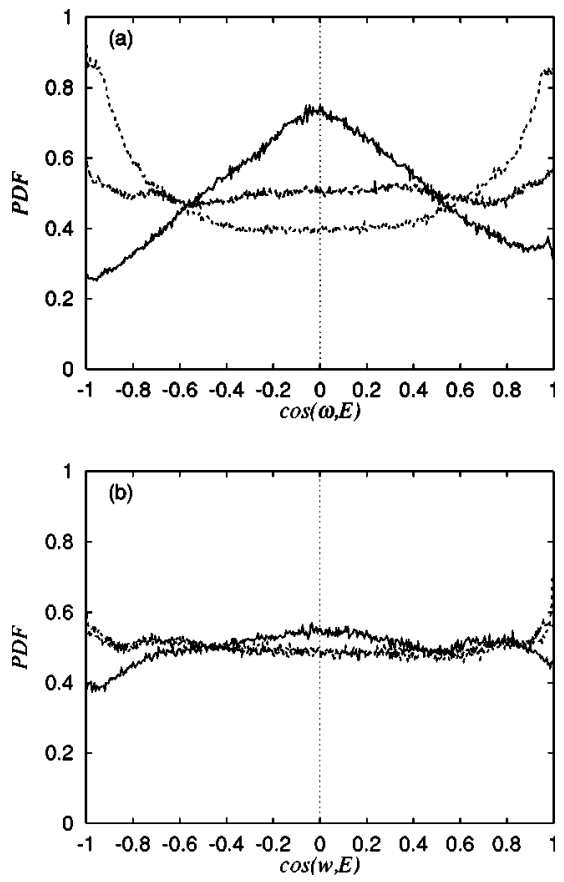

FIG. 10. Alignment of (a) $\boldsymbol{\omega}$ and (b) $\boldsymbol{w}$ with eigenvectors of the Cauchy-Green tensor $\boldsymbol{J}^{T} \boldsymbol{J}, \quad \boldsymbol{E}_{\alpha}$ (solid), $\boldsymbol{E}_{\beta}$ (dashed), and $\boldsymbol{E}_{\gamma}$ (dotted).

and their product is unity $\alpha \beta \gamma=1$. There are orthogonal eigenvectors $\boldsymbol{E}_{\alpha}, \boldsymbol{E}_{\beta}, \boldsymbol{E}_{\gamma}$ associated with them.

In view of Eq. (4.8) we need to consider alignment of $\boldsymbol{\omega}_{0}$ with $\boldsymbol{E}_{\alpha}, \boldsymbol{E}_{\beta}$, or $\boldsymbol{E}_{\gamma}$ to estimate the enstrophy growth. This alignment problem inherently dictates the influence of the initial condition on the enstrophy evolution later on. To study this alignment problem we need to evaluate the initial vorticity for each fluid particle. But it is not easy to track quite a large number of fluid particles accurately. Here, to avoid the difficulty associated with particle tracking we simply invert the Jacobian matrix, whose determinant is $1(\neq 0)$, as

$$
\boldsymbol{\omega}_{0}(\boldsymbol{a})=\boldsymbol{J}^{-1} \boldsymbol{\omega}(\boldsymbol{a}, t)
$$

Likewise similar to Eq. (4.8) we can write for material line elements (that is, the passive vectors in the case of inviscid flows)

$$
|\boldsymbol{w}(\boldsymbol{a}, t)|^{2}=\boldsymbol{w}_{0}^{T}(\boldsymbol{a}) \boldsymbol{J}^{T} \boldsymbol{J} \boldsymbol{w}_{0}(\boldsymbol{a}) .
$$

In Fig. 10(a) we show the PDF of the cosines of angles between $\boldsymbol{E}_{\alpha}, \boldsymbol{E}_{\beta}$, and $\boldsymbol{E}_{\gamma}$ with $\boldsymbol{\omega}$ at $t=0.75$. The PDFs show that $\boldsymbol{\omega}$ has a tendency to be parallel to $\boldsymbol{E}_{\gamma}$ and it also has a tendency to be perpendicular to $\boldsymbol{E}_{\alpha}$. Thus this method captures another aspect of depleted nonlinearity in that, it shows that intense vorticity is correlated with the most compressing eigenvector of the Cauchy-Green tensor. For $\boldsymbol{E}_{\beta}$ direction, no clear characteristic feature is observed. We should, however, keep in mind that this alignment feature is not so strong as the one observed in the conventional alignment analysis in Figs. 2 and 3 (compare the ordinate axis in Figs. 10). In Fig. 10 (b) we show the PDF of the cosines of angles between $w$ 


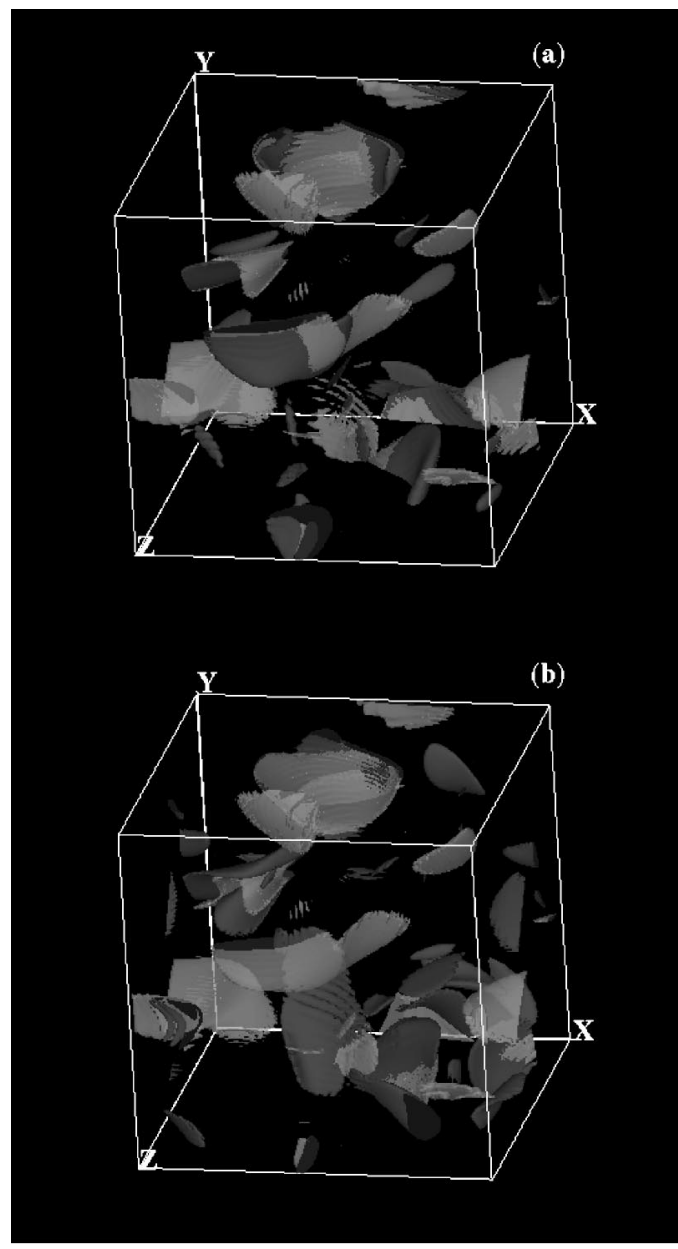

FIG. 11. Comparison of isosurface plots of $\operatorname{tr}\left(\boldsymbol{J}^{T} \boldsymbol{J}\right)$ (lighter) with (a) $|\boldsymbol{\omega}|^{2}$ (darker) and (b) $|\boldsymbol{w}|^{2}$ (darker). The thresholds chosen are $0.2\left(\max |\boldsymbol{\omega}|^{2}\right), 0.2\left(\max |\boldsymbol{w}|^{2}\right)$, and $0.3\left(\max \left[\operatorname{tr}\left(\boldsymbol{J}^{T} \boldsymbol{J}\right)\right]\right)$.

and $\boldsymbol{E}_{\alpha}, \boldsymbol{E}_{\beta}$, and $\boldsymbol{E}_{\gamma}$ at the same time. The PDFs show almost uniform distributions in all the three directions, making a sharp contrast to the case of vorticity. Therefore, in the analysis of the alignment problem associated with the Cauchy-Green tensor, depletion of nonlinearity can be observed more clearly than in that associated with the rate-ofstrain tensor.

In Fig. 11(a) we compare the isosurfaces of $|\boldsymbol{\omega}|^{2}$ with those of $\operatorname{tr}\left(\boldsymbol{J}^{T} \boldsymbol{J}\right)$. Layerlike structures are predominant in both fields and the locations are overlapping. In Fig. 11(b) the isosurface of $|\boldsymbol{w}|^{2}$ is compared with that of $\operatorname{tr}\left(\boldsymbol{J}^{T} \boldsymbol{J}\right)$. The regions with intense $|\boldsymbol{w}|^{2}$ are again layerlike but they do not appear to overlap with the regions with $\operatorname{large} \operatorname{tr}\left(\boldsymbol{J}^{T} \boldsymbol{J}\right)$. However, unfortunately we have at present no explanation for it.

Finally, a comparison of the two alignment problems is in order. The conventional alignment is instantaneous and mostly kinematic, whereas the alignment problem considered here is cumulative and dynamical in that it dictates finitetime evolution. The method described here may be regarded as a first step toward extracting Lagrangian information and should be applied for various fluid flows.

\section{SUMMARY AND DISCUSSION}

In order to characterize the effect of nonlinear constraint imposed by the Biot-Savart formula, we have compared time evolution of the vorticity and passive vectors in detail using numerical simulations of Navier-Stokes turbulence.

For finite- (or, long-) time evolution, we find that the PVs are stretched more intensely than the vorticity. A number of quantitative evidences are available. First, as far as the present numerical simulations which cover up to $R_{\lambda} \sim 100$ are concerned, $\nu\left\langle|w|^{2}\right\rangle$ keeps growing with decreasing viscosity, whereas $\nu\left\langle|\boldsymbol{\omega}|^{2}\right\rangle$ appears to be limited in growth. Second, the PDF of the stretching rate of PVs is shifted for more intense stretching compared with that of the vorticity.

Using various initial conditions, we find for a majority class of passive vectors

$$
\left\langle|\boldsymbol{w}(\boldsymbol{x}, t)|^{2}\right\rangle>\left\langle|\boldsymbol{\omega}(\boldsymbol{x}, t)|^{2}\right\rangle
$$

in the long run. But, this inequality does not necessarily hold valid at all times for certain initial conditions.

The point is made clear by considering infinitesimal- (i.e., short-) time evolution. We have investigated the PDFs of the growth rates of the PV variance

$$
\sigma_{\mathrm{PV}}(t)=\frac{1}{\left\langle|\boldsymbol{w}|^{2}\right\rangle} \frac{d}{d t}\left\langle|\boldsymbol{w}|^{2}\right\rangle=2 \frac{\langle\boldsymbol{w} \cdot \boldsymbol{S} \cdot \boldsymbol{w}\rangle}{\left\langle|\boldsymbol{w}|^{2}\right\rangle}
$$

It is found that the PDF is close to a Gaussian distribution, even when the samples are taken around the vorticity, which is a solution of the Navier-Stokes equations. This implies that there are infinitely many PVs that grow more slowly than the vorticity.

Then, the apparent dominance of the growth of PVs over that of the vorticity is a consequence of correlation built up between the vorticity and the rate of strain over finite-time evolution. It is difficult to get a proper estimate of the vortex stretching term by purely kinematic methods, that is, by handling the Biot-Savart law only.

For treating finite-time evolution, use of a kind of alignment problem of the Cauchy-Green tensor is proposed. As a first step, we have tested it for the Euler equations and observed that it is found that vorticity tends to align itself with the most compressing eigenvector of the Cauchy-Green tensor. In this sense this method captures depletion of nonlinearity clearly and its detailed application may be useful for the characterization of flow structure.

It may be useful to state physical meanings of passive vector $\boldsymbol{w}$ here. One is the kinematic magnetohydrodynamics (MHD) equations. This is obtained by dropping the Lorentz force term $(\boldsymbol{B} \cdot \boldsymbol{\nabla}) \boldsymbol{B}$ and replacing $\boldsymbol{B}$ by $\boldsymbol{w}$ in the usual set of MHD equations,

$$
\begin{gathered}
\frac{\partial \boldsymbol{u}}{\partial t}+(\boldsymbol{u} \cdot \boldsymbol{\nabla}) \boldsymbol{u}=-\boldsymbol{\nabla} p+(\boldsymbol{B} \cdot \boldsymbol{\nabla}) \boldsymbol{B}+\nu \triangle \boldsymbol{u}, \\
\frac{\partial \boldsymbol{B}}{\partial t}+(\boldsymbol{u} \cdot \boldsymbol{\nabla}) \boldsymbol{B}=(\boldsymbol{B} \cdot \boldsymbol{\nabla}) \boldsymbol{u}+\nu \triangle \boldsymbol{B} .
\end{gathered}
$$


The other one is the material line elements for the case $\nu$ $=0$.

As mentioned above, the spatial resolution employed in this work is not very high, particularly in the production of the PDFs of the stretching rates of PVs. It is of interest to examine whether the results may change qualitatively or not in turbulence at higher Reynolds numbers.

\section{ACKNOWLEDGMENTS}

The author thanks Professor A. Tsinober and Professor T. Ishihara for useful comments on the draft of this paper. This work has been partially supported by a grant-in-aid for scientific research from the Ministry of Education, Culture, Sports, Science and Technology of Japan, under Grant Nos. 11304005 and 11214204.

\section{APPENDIX A: TWO-DIMENSIONAL NAVIER-STOKES EQUATIONS}

In two dimensions, a similar active-passive comparison is well known (see, for example, $[18,19])$. In this case vorticity $\omega$ and a passive scalar $\theta$ satisfy the same form of equations,

$$
\frac{\partial \omega}{\partial t}+(\boldsymbol{u} \cdot \nabla) \omega=\nu \triangle \omega
$$

and

$$
\frac{\partial \theta}{\partial t}+(\boldsymbol{u} \cdot \boldsymbol{\nabla}) \theta=\nu \triangle \theta
$$

where $\boldsymbol{\nabla} \cdot \boldsymbol{u}=0$.

The dissipation rate of the enstrophy is given by

$$
\eta=-\frac{d}{d t}\left\langle\frac{\omega^{2}}{2}\right\rangle=-\nu\left\langle|\nabla \omega|^{2}\right\rangle
$$

and that of passive scalar variance by

$$
\eta_{\mathrm{PS}}=-\frac{d}{d t}\left\langle\frac{\theta^{2}}{2}\right\rangle=\nu\left\langle|\nabla \theta|^{2}\right\rangle
$$

Numerical simulations starting from a random initial condition, which is localized in the low wave number components, are shown in Fig. 12 for three different values of viscosity $\nu=1 \times 10^{-4}, \quad 2 \times 10^{-4}, \quad 4 \times 10^{-4}$. Unlike the three-dimensional (3D) counterpart (Figs. 1), not only $\nu\left\langle|\nabla \omega|^{2}\right\rangle$ but also $\nu\left\langle|\nabla \theta|^{2}\right\rangle$ depends on viscosity only weakly; the former changes by about $28 \%$ and the latter by $24 \%$ when the value of viscosity changes by a factor of 4 . This may be interpreted as follows. Not only Eq. (A1) but also Eq. (A2) possesses a positive-definite conserved quantity; there are two inviscid conservation laws $\left\langle\omega^{2}\right\rangle$ and $\left\langle\theta^{2}\right\rangle$ underlying Eqs. (A1) and (A2). These two conserved quantities give $\eta$ and $\eta_{\mathrm{PV}}$ physical meanings (see Table III) as their rates of dissipation.

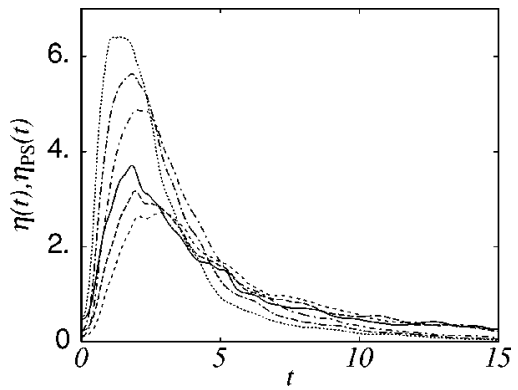

FIG. 12. An example of active-passive comparison: time evolution of the enstrophy dissipation rate $\eta(t)$ (the three curves above) and a corresponding quantity $\eta_{\mathrm{PS}}(t)$ (the three curves below) for passive scalars.

\section{APPENDIX B: A SPECIAL CASE}

An example can be constructed where a class of passive vectors $\boldsymbol{w}$ grows faster than the vorticity $\boldsymbol{\omega}$ in the early stage by making use of the Taylor-Green vortex. We take

$$
\boldsymbol{\omega}=\left(\begin{array}{l}
(C-B) \sin x \cos y \cos z \\
(A-C) \cos x \sin y \cos z \\
(B-A) \cos x \cos y \sin z
\end{array}\right)
$$

and

$$
\boldsymbol{w}=\left(\begin{array}{c}
(R-Q) \sin (x+\phi) \cos (y+\phi) \cos (z+\phi) \\
(P-R) \cos (x+\phi) \sin (y+\phi) \cos (z+\phi) \\
(Q-P) \cos (x+\phi) \cos (y+\phi) \sin (z+\phi)
\end{array}\right)
$$

where $A, B, C$ and $P, Q, R$ are amplitudes of the vorticity and a passive vector, respectively. In this case we can see easily that

$$
\frac{d}{d t}\left\langle\frac{|\boldsymbol{\omega}|^{2}}{2}\right\rangle=\frac{d}{d t}\left\langle\frac{|\boldsymbol{w}|^{2}}{2}\right\rangle=0 .
$$

In general, the following formulas can be derived for the second time derivatives of the enstrophy (-like) quantities:

$$
\frac{d^{2}}{d t^{2}}\left\langle\frac{|\boldsymbol{\omega}|^{2}}{2}\right\rangle=\langle(\boldsymbol{S} \cdot \boldsymbol{\omega}) \cdot(\boldsymbol{S} \cdot \boldsymbol{\omega})\rangle-\langle\boldsymbol{\omega} \cdot \boldsymbol{P} \cdot \boldsymbol{\omega}\rangle
$$

and

TABLE III. Relationship between positive-definite conserved quantities and depletion of nonlinearity.

\begin{tabular}{llll}
\hline \hline & Active & Passive & \\
\hline 3D & $\left\langle|\boldsymbol{u}|^{2}\right\rangle$ & $\langle\boldsymbol{u} \cdot \delta \boldsymbol{l}\rangle$ & $\nu\left\langle|\boldsymbol{\omega}|^{2}\right\rangle$ is meaningful \\
& $\langle\boldsymbol{u} \cdot \boldsymbol{\omega}\rangle$ & & $\nu\left\langle|\delta \boldsymbol{l}|^{2}\right\rangle$ is meaningless \\
2D & $\left\langle|\boldsymbol{u}|^{2}\right\rangle$ & $\left\langle\theta^{2}\right\rangle$ & $\nu\left\langle|\boldsymbol{\nabla} \omega|^{2}\right\rangle$ is meaningful \\
& $\left\langle\omega^{2}\right\rangle$ & & $\nu\left\langle|\boldsymbol{\nabla} \theta|^{2}\right\rangle$ is meaningful \\
\hline \hline
\end{tabular}




$$
\begin{aligned}
\frac{d^{2}}{d t^{2}}\left\langle\frac{|\boldsymbol{w}|^{2}}{2}\right\rangle= & \langle(\boldsymbol{S} \cdot \boldsymbol{w}) \cdot(\boldsymbol{S} \cdot \boldsymbol{w})\rangle-\langle\boldsymbol{w} \cdot \boldsymbol{P} \cdot \boldsymbol{w}\rangle+\frac{|\boldsymbol{\omega} \times \boldsymbol{w}|^{2}}{4} \\
& +(\boldsymbol{\omega} \times \boldsymbol{w}) \cdot \boldsymbol{S} \cdot \boldsymbol{w}
\end{aligned}
$$

We have evaluated these by using computer algebra MAPLEV after eliminating $C$ and $R$ using $C=-(A+B)$ and $R=$ $-(P+Q)$. The final results are

$$
\frac{d^{2}}{d t^{2}}\left\langle\frac{|\boldsymbol{\omega}|^{2}}{2}\right\rangle=\frac{5}{64}\left(A^{2}+A B+B^{2}\right)^{2}
$$

and

$$
\begin{aligned}
\frac{d^{2}}{d t^{2}}\left\langle\frac{|\boldsymbol{w}|^{2}}{2}\right\rangle= & -\frac{1}{32}\left(10 A^{2} P Q+7 B^{2} P^{2}+7 A^{2} Q^{2}+10 B^{2} Q^{2}\right. \\
& +10 B^{2} P Q+16 Q B A P+10 A^{2} P^{2}+10 P^{2} B A \\
& \left.+10 Q^{2} B A\right) \cos ^{4}(\phi)+\frac{1}{32}\left(8 Q^{2} B A+8 B^{2} Q^{2}\right. \\
& +8 A^{2} P Q+2 Q B A P+11 B^{2} P^{2}+8 P^{2} B A \\
& \left.+11 A^{2} Q^{2}+8 B^{2} P Q+8 A^{2} P^{2}\right) \cos ^{2}(\phi) \\
& +\frac{1}{128}\left(18 P^{2} B A+9 B^{2} P^{2}+18 Q^{2} B A\right. \\
& +18 B^{2} P Q+36 Q B A P+18 A^{2} P Q+18 B^{2} Q^{2} \\
& \left.+9 A^{2} Q^{2}+18 A^{2} P^{2}\right) .
\end{aligned}
$$

The condition that they share their Fourier spectrum is

$$
A^{2}+A B+B^{2}=P^{2}+P Q+Q^{2} .
$$

By introducing a set of new variables

$$
\begin{aligned}
& A=(a-b) / \sqrt{2}, \quad B=(a+b) / \sqrt{2}, \\
& P=(p-q) / \sqrt{2}, \quad Q=(p+q) / \sqrt{2},
\end{aligned}
$$

the above condition is reduced to

$$
3 a^{2}+b^{2}=3 p^{2}+q^{2}
$$

So, the amplitudes of the passive vector can be parametrized as

$$
\begin{aligned}
& p=\sqrt{3 a^{2}+b^{2}} \cos \theta, \\
& q=\sqrt{3 a^{2}+b^{2}} \sin \theta .
\end{aligned}
$$

Using them we find

$$
\frac{d^{2}}{d t^{2}}\left\langle\frac{|\boldsymbol{w}|^{2}}{2}\right\rangle-\frac{d^{2}}{d t^{2}}\left\langle\frac{|\boldsymbol{\omega}|^{2}}{2}\right\rangle \geqslant 0,
$$

if $\cos ^{2} \phi \geqslant 1-(\sqrt{30} / 6)=0.91$. Therefore for the class (B2) around Eq. (B1), PVs grow more intensely than the vorticity in the initial stage of development, if $|\phi|$ is not close to $\pi / 2$.
[1] U. Frisch, Turbulence (Cambridge University Press, Cambridge, 1995).

[2] This expectation has been controversial; see, e.g., [5].

[3] R. H. Kraichnan and R. Panda, Phys. Fluids 31, 2395 (1988).

[4] A. Tsinober, L. Shtilman, and H. Vaisburd, Fluid Dyn. Res. 21, 477 (1997).

[5] A. Tsinober, Eur. J. Mech. B/Fluids 17, 421 (1998).

[6] A. Tsinober, M. Ortenberg, and L. Shtilman, Phys. Fluids 11, 2291 (1999).

[7] P. Constantin and C. Fefferman, Indiana Univ. Math. J. 42, 775 (1993).

[8] P. Constantin, C. Fefferman, and A. Majda, Commun. Partial Differ. Equ. 21, 559 (1996).

[9] P. Constantin, SIAM Rev. 36, 73 (1994).

[10] K. Ohkitani, J. Phys. Soc. Jpn. 67, 44 (1998).
[11] In [10] the notation $\boldsymbol{\delta} l$ was used for $\boldsymbol{w}$.

[12] S. S. Girmiaji and S. B. Pope, J. Fluid Mech. 220, 427 (1990).

[13] M.-J. Huang, Phys. Fluids 8, 2203 (1996).

[14] A. Tsinober, Actes du $12^{e}$ Congrès Francais de Mécanique 3, 409 (1995).

[15] S. Kida and S. Goto, Phys. Fluids 14, 352 (2002).

[16] R. H. Kraichnan and Y. Kimura, Prog. Astronaut. Aeronaut. 162, 19 (1994).

[17] J. M. Ottino, The Kinematics of Mixing: Stretching, Chaos, and Transport (Cambridge University Press, Cambridge, 1989).

[18] A. Babiano, C. Basdevant, B. Legras, and R. Sadourny, J. Fluid Mech. 183, 379 (1987).

[19] K. Ohkitani, Phys. Fluids A 3, 1598 (1991). 